\title{
ESTUDO DA SECAGEM DE CHIPS DE BERINJELA EM TÚNEL DE VENTO
}

\author{
L. N. PINHEIRO ${ }^{1}$, D. BEGROW ${ }^{1}$, M. B. RAVAZIO ${ }^{1}$ e L. de M. TERRA ${ }^{1}$ \\ ${ }^{1}$ Universidade Federal de Santa Maria, Faculdade de Engenharia Química \\ E-mail para contato: np.luiza@gmail.com
}

\begin{abstract}
RESUMO - A berinjela é um alimento funcional em virtude da presença dos antioxidantes, antocianinas, o que a torna interessante do ponto de vista nutricional. Nesse contexto, novas formas de consumo do alimento podem ser obtidas via secagem, que proporciona maior vida de prateleira e transporte e armazenamento facilitados. O túnel de vento como equipamento traz maior eficiência ao envolver a convecção forçada como mecanismo de troca de calor. A cinética de secagem e a modelagem do processo foram analisadas visando a otimização do mesmo. O modelo de melhor ajuste foi o modelo Midilli et al, com coeficiente de correlação de 0,9996. Quanto à secagem, foram obtidos três modelos significativos para as variáveis redução de massa, redução de atividade de água e atividade de água final em função de temperatura, velocidade e tempo codificados. A otimização para o processo em túnel de vento deu-se para temperatura de $61,9^{\circ} \mathrm{C}$, velocidade de $0,575 \mathrm{~m} / \mathrm{s}$ e tempo de 150 minutos.
\end{abstract}

\section{INTRODUÇÃO}

A berinjela (Solanum melongena) é um fruto nutricionalmente interessante devido aos seus altos teores de fibra alimentar, de minerais e de antioxidantes, que atuam na redução do colesterol. Um exemplo são as antocianinas, responsáveis pela coloração roxa da casca e pela classificação como alimento funcional (SANTOS et al, 2002). Obter um alimento seco de berinjela é uma alternativa para o consumo da mesma. A retirada da água é uma parte integrante do processamento de diversos alimentos, sendo os principais objetivos da desidratação o prolongamento da vida de prateleira, diminuição dos custos de manuseio e transporte, pois se têm a redução do peso e da atividade de água que resulta em menor atividade de microrganismos (BORGES et al, 2008). Define-se secagem como retirada de água livre de um material mediante evaporação, envolvendo fenômenos de transporte de massa e energia (MCMINN e MAGEE, 1999). A transferência de calor ocorre devido a diferença de temperaturas entre o alimento e o ar aquecido do aparelho, enquanto a de massa é resultado da diferença da pressão parcial de vapor da corrente gasosa e do alimento, o que propicia o arraste do vapor do alimento (PARK et al, 2001). Dessa forma, objetivou-se a elaboração de um processo de secagem de chips de berinjela em túnel de vento otimizado, por meio da análise cinética e modelagem da secagem.

\section{MATERIAIS E MÉTODOS}

As matérias primas utilizadas foram berinjelas de coloração roxa escura, obtidas no mercado local de Santa Maria, RS, baseando-se em aparência saudável e coloração constante e uniforme da casca. Elas foram lavadas e cortados longitudinalmente com auxílio de um descascador, fazendo-se uso da casca, em virtude da mesma ser rica em antocianinas, caracterizando o aspecto funcional do alimento. Foram usadas amostras de 14 a 15 gramas, totalizando, aproximadamente, 5 chips. Foi realizada a desidratação 


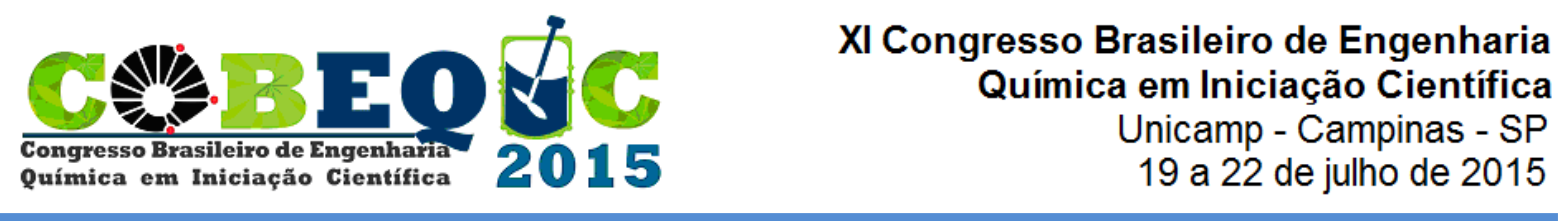

osmótica (DO) como pré-tratamento de secagem, a qual é um procedimento vantajoso, pois proporciona uma perda de água de 40 a 70\%, amenizando as condições de secagem posterior (RAUOLT-WACK, 1994). O agente osmótico usado foi uma solução de $200 \mathrm{~g}$ a $46,45^{\circ}$ Brix de sacarose e sal, sendo apenas $3 \%$ da massa de sólidos composta por sal. A DO, previamente otimizada, foi conduzida em banho termostático provido de agitação em uma temperatura de $36,45^{\circ} \mathrm{C}$ por 30 minutos (PINHEIRO et al, 2014).

\subsection{Cinética de Secagem}

A análise da cinética de secagem em túnel de vento foi feita considerando-se a variação dos fatores temperatura e velocidade do ar, usando-se uma faixa de 30 a $70^{\circ} \mathrm{C}$ e 0,5 a $2 \mathrm{~m} / \mathrm{s}$, baseando-se em Ertekin e Yaldiz (2004) e objetivou a obtenção de uma faixa temporal para a operação de secagem dos chips de berinjela. Assim, elaborou-se um planejamento experimental com cinco valores de cada variável, combinando-os. A secagem foi realizada em secador convectivo do tipo túnel de vento. As amostras foram pesadas in natura e submetidas a operação, com pesagens de meia em meia hora, até obtenção de massa constante três vezes consecutivas. Nesse instante, considerou-se que a umidade de equilíbrio foi atingida e que o processo de secagem estava encerrado. Os experimentos foram realizados em duplicata. Para analisar os dados, foram realizados ajustes aos modelos indicados pela Tabela 1, utilizando o software Polymath® (RADÜNZ et al, 2010). A escolha do melhor modelo foi feita com base no valor do coeficiente de correlação $\left(\mathrm{R}^{2}\right)$. Esses modelos são todos empíricos ou semi-empíricos, de modo que os parâmetros de ajuste não têm significado físico e tem-se o tempo como variável independente e o adimensional razão de umidade (RU) como dependente, além de outras variáveis usadas como parâmetros. O RU é obtido com base na Equação 1. A variável U indica a umidade em base seca, sendo $U_{i}$ a umidade em cada instante, $U_{e}$ a umidade de equilíbrio (umidade quando se atinge massa constante) e $\mathrm{U}_{0}$ a umidade da amostra in natura. No caso, a umidade $\mathrm{U}_{\mathrm{i}}$ é calculada conforme a Equação 2, sendo baseada na diferença da massa da amostra no instante i e da massa seca final (produto da massa in natura pela porcentagem de sólidos da berinjela 8,715\%, simbolizado por MSF), dividida pela massa seca final.

$$
\begin{gathered}
R U_{i}=\frac{U_{i}-U_{e}}{U_{0}-U_{e}} \\
U_{i}=\frac{M_{i}-M S F}{M S F}
\end{gathered}
$$

\subsection{Análise da Influência das Variáveis de Secagem}

Para realização da modelagem da secagem, foram realizados 12 experimentos conforme planejamento experimental do tipo delineamento composto central rotacional (DCCR) para três variáveis. Assim, utilizaram-se 8 pontos fatoriais, 6 pontos axiais (para codificação $\pm 1,68$ ) e 4 pontos centrais (RODRIGUES e IEMMA, 2009). Foram analisados o efeito da temperatura (faixa de 30 a $\left.70^{\circ} \mathrm{C}\right)$, da velocidade do ar $(0,5$ a $2 \mathrm{~m} / \mathrm{s})$ e do tempo (150 a 540 minutos), sendo a faixa temporal obtida com base nos resultados da cinética de secagem. No caso, a codificação foi realizada considerando-se os máximos como 1,68 e os mínimos como -1,68. Como variáveis dependentes, analisou-se a massa final (MF), redução de massa (RM), atividade de água final (AAF) e redução de atividade de água (RAA), com a atividade de água determinada pelo equipamento Aqualab ${ }^{\circledR}$. As análises foram realizadas no 


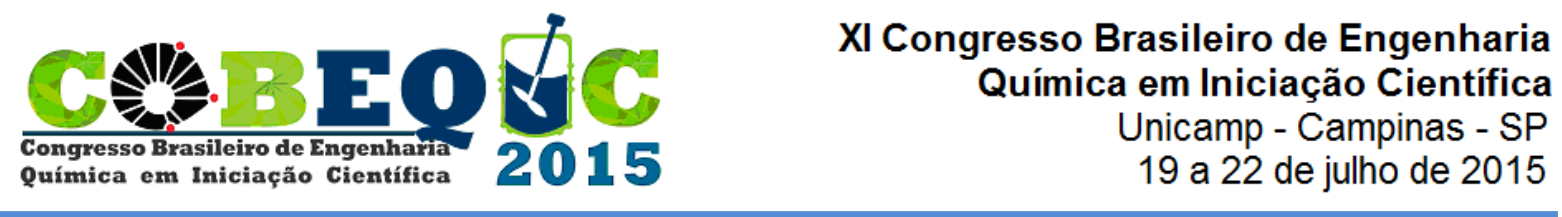

software Statistica ${ }^{\circledR}$ considerando significância de $95 \%$, sendo considerado para a avaliação do modelo o coeficiente de determinação $\left(\mathrm{R}^{2}\right)$ e o erro médio relativo do modelo. O erro médio é dado pela média aritmética do quociente entre o resíduo e valor experimental da variável. Os parâmetros considerados significativos nos modelos são aqueles que apresentarem p-valor inferior a 0,05 . Os modelos foram elaborados com base na temperatura, velocidade e tempo codificados. Após a obtenção dos modelos, foi realizada uma otimização da operação, considerando a região de maior perda de massa e a região de maior redução de atividade de água e de atividade de água final mínima e a sobreposição dessas regiões.

Tabela 1 - Modelos para ajuste da cinética de secagem

\begin{tabular}{cc}
\hline Modelo & Equação \\
\hline Midilli et al & $\mathrm{RU}=\mathrm{a} \cdot \exp (-\mathrm{k} \cdot \mathrm{tn})+\mathrm{b} \cdot \mathrm{t}$ \\
Aproximação da Difusão & $\mathrm{RU}=\mathrm{a} \cdot \exp (-\mathrm{k} \cdot \mathrm{t})+(1-\mathrm{a}) \cdot \exp (-\mathrm{k} \cdot \mathrm{b} \cdot \mathrm{t})$ \\
Page & $\mathrm{RU}=\exp (-\mathrm{k} \cdot \mathrm{tn})$ \\
Logaritmo & $\mathrm{RU}=\mathrm{a} \cdot \exp (-\mathrm{k} \cdot \mathrm{t})+\mathrm{c}$ \\
Henderson e Pabis & $\mathrm{RU}=\mathrm{a} \cdot \exp (-\mathrm{k} \cdot \mathrm{t})$ \\
Page Modificada & $\mathrm{RU}=\exp \left[(-\mathrm{k} \cdot \mathrm{t})^{\mathrm{n}}\right]$ \\
Dois Termos & $\mathrm{RU}=\mathrm{a} \cdot \exp (-\mathrm{k} \cdot \mathrm{t})+\mathrm{b} \cdot \exp (-\mathrm{q} \cdot \mathrm{t})$ \\
Exponencial de Dois Termos & $\mathrm{RU}=\mathrm{a} \cdot \exp (-\mathrm{k} \cdot \mathrm{t})+(1-\mathrm{a}) \cdot \exp (-\mathrm{k} \cdot \mathrm{a} \cdot \mathrm{t})$ \\
Newton & $\mathrm{RU}=\exp (-\mathrm{k} \cdot \mathrm{t})$ \\
Henderson e Pabis Modificada & $\mathrm{RU}=\mathrm{a} \cdot \exp (-\mathrm{k} \cdot \mathrm{t})+\mathrm{b} \cdot \exp (-\mathrm{q} \cdot \mathrm{t})+\mathrm{c} \cdot \exp (-\mathrm{w} \cdot \mathrm{t})$ \\
Wangh e Singh & $\mathrm{RU}=1+\mathrm{a} \cdot \mathrm{t}+\mathrm{b} \cdot \mathrm{t} 2$ \\
\hline
\end{tabular}

\section{RESULTADOS E DISCUSSÕES}

\subsection{Cinética de Secagem}

O melhor ajuste foi obtido para o modelo Midilli et al, o qual apresentou um coeficiente de correlação de 0,9996 sendo este resultado confirmado pelos trabalhos de Ertekin e Yaldiz (2004). As curvas cinéticas estão apresentadas na Figura 1, havendo uma curva para cada situação testada, totalizando 9 curvas, comparando-se os pontos experimentais e o modelo ajustado. Outros modelos que obtiveram bons ajustes foram Lewis, Aproximação da Difusão, Exponencial de Dois Termos, Henderson e Pabis modificado e Page Modificada, com $\mathrm{R}^{2}$ de 0,9969; 0,9985; 0,9990; 0,9976 e 0,9969, respectivamente. Além disso, com base nos experimentos de cinética de secagem, obteve-se os tempos mínimos e máximos de operação, 150 e 540 minutos, usados para elaboração do DCCR citado anteriormente.

\subsection{Análise da Influência das Variáveis de Secagem}

Com base nos valores experimentais, foram obtidos 3 modelos considerados significativos $\left(\mathrm{R}^{2}>0,7000\right)$, os quais são dados pelas Equações de 3 a 5 . O valor entre parênteses após cada termo indica o $\mathrm{p}$-valor da variável envolvida. As variáveis independentes temperatura $(\mathrm{T})$, velocidade do $\operatorname{ar}(\mathrm{v})$ e tempo (t) são em valores codificados, o que é vantajoso para a formulação matemática do modelo 
(RODRIGUES e IEMMA, 2009). No caso, para a variável massa final (MF), o modelo obtido apresentou $\mathrm{R}^{2}$ de 0,2425 , inviabilizando-o.

$$
\begin{aligned}
& R M=4,68+0,06 T(0,73)-0,11 T^{2}(0,53)-0,003 v(0.99)+0,09 v^{2}(0,60)-0,36 t(0.053)+ \\
& 0,08 t^{2}(0,66)-0,64 T v(0,02)-0,34 T t(0,14)-0,15 t v(0,50) \\
& R A A=0,426+0,034 T(0,01)-0,039 T^{2}(0,01)-0,001 v(0,95)-0,010 v^{2}(0,38)+0,017 t(0,14)- \\
& 0,003 t^{2}(0,77)-0,009 T v(0,53)-0,009 T t(0,54)-0,003 v t(0,86) \\
& A A F=0,563-0,034 T(0,01)+0,039 T^{2}(0,01)+0,001 v(0,95)+0,010 v^{2}(0,38)-0,017 t(0,14)+ \\
& 0,003 t^{2}(0,77)+0,009 T v(0,53)+0,009 T t(0,54)+0,003 v t(0,86)
\end{aligned}
$$

Figura 1 - Dados ajustados ao modelo Midilli et al

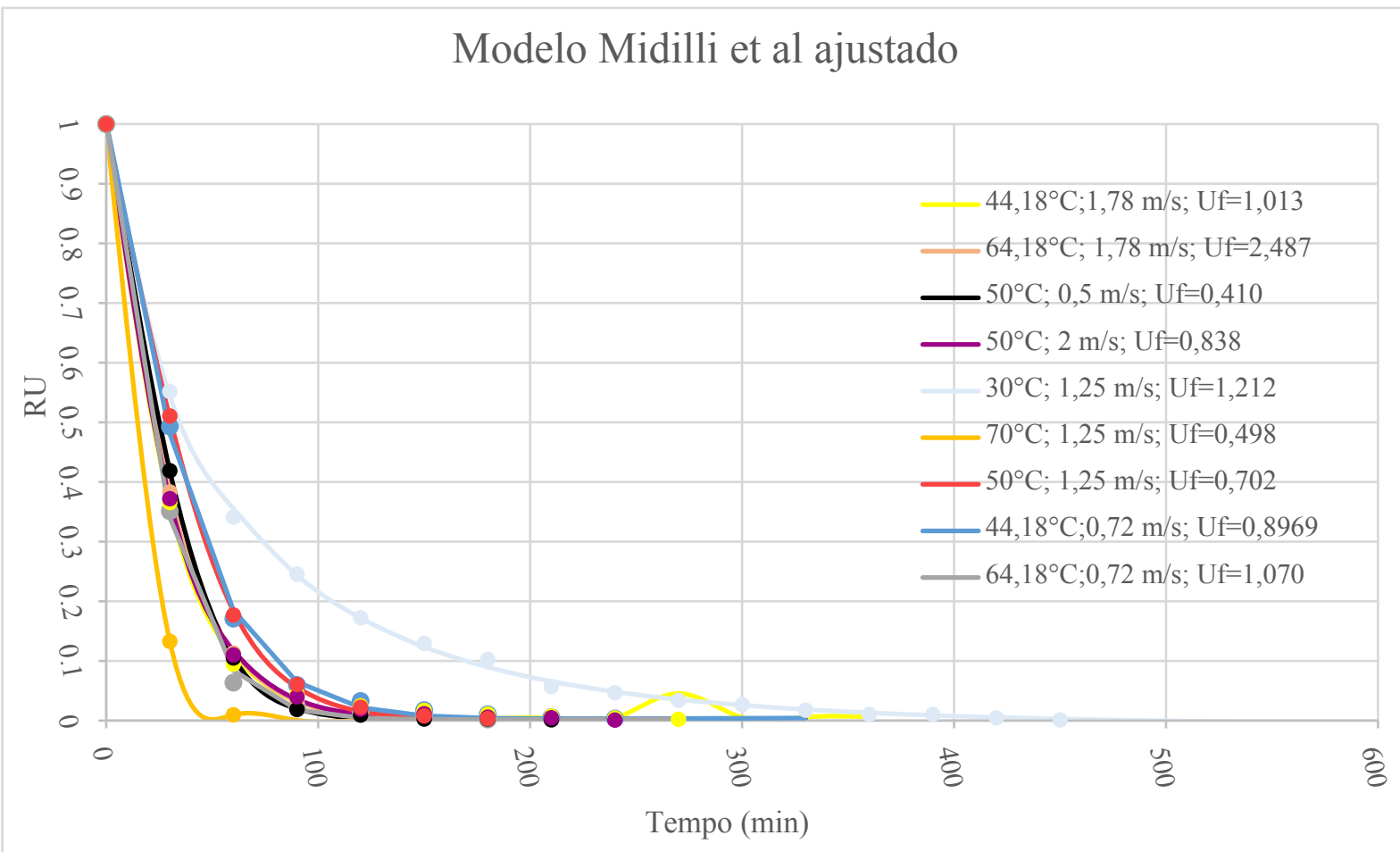

O modelo obtido para a redução da massa apresentou $\mathrm{R}^{2}$ igual a 0,7048 estando na região limítrofe de aceitação, sendo seu erro médio relativo de 6,68 \% e parâmetro significativo apenas a interação entre a temperatura e velocidade, com p-valor igual a 0,015 . No caso o efeito deste parâmetro é negativo, indicando que uma combinação adequada de temperatura e velocidade permite uma redução de massa máxima. Tem-se a representação gráfica deste modelo na Figura 2, na qual se percebe a região de otimização nas extremidades, uma combinação com temperatura superior a $61,9^{\circ} \mathrm{C}$ e velocidade inferior a $0,8 \mathrm{~m} / \mathrm{s}$ ou temperatura inferior a $44,05^{\circ} \mathrm{C}$ e velocidade superior a $1,7 \mathrm{~m} / \mathrm{s}$. Havendo, portanto 


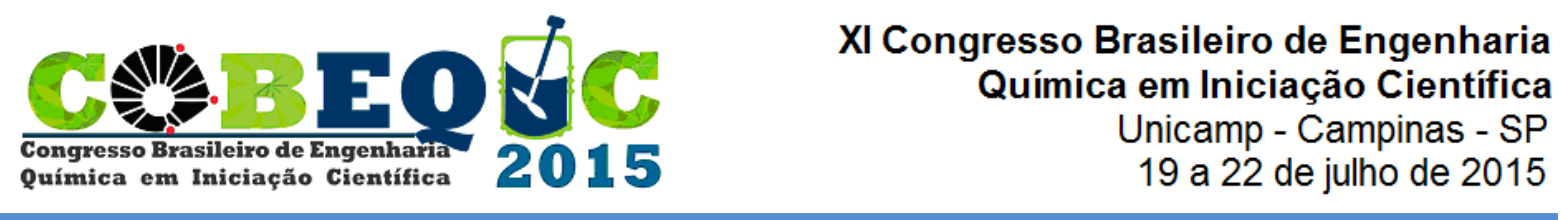

um balanço entre as grandezas das variáveis. Para a redução de atividade de água obteve-se $\mathrm{R}^{2}$ igual a 0,7689 e erro relativo de $6,59 \%$, apresentando melhores características que o modelo anterior. Contudo, obteve-se apenas a temperatura como parâmetro significativo, sendo o p-valor para as contribuições linear e quadrática, 0,012 e 0,008, respectivamente. Como o efeito quadrático apresenta valor negativo, tem-se novamente um valor de temperatura que permite um máximo de redução de atividade de água. Com base nos gráficos da Figura 3, o ponto ótimo de temperatura pode ser considerado entre 50 e $61,9^{\circ} \mathrm{C}$. Por fim, para a atividade de água final, o modelo obtido apresentou $\mathrm{R}^{2}$ igual a 0,7689 e erro relativo de 3,9\%. No caso, comparando-se as Equações 4 e 5, percebe-se que com exceção do parâmetro independe, os outros apresentam o mesmo módulo, com sinais contrários, de modo que o gráfico apresentado é o mesmo, apenas invertendo-se as regiões de máximo e mínimo, como pode ser verificado na Figura 4, que relaciona temperatura e velocidade. Para este modelo, novamente apenas a temperatura foi significativa, com p-valor para contribuição linear e quadrática de 0,021 e 0,022 , respectivamente. Sendo assim, tem-se a região de mínima atividade de água final para uma temperatura entre 50 e $61,9^{\circ} \mathrm{C}$. Dessa forma, realizando uma otimização geral da secagem de chips de berinjela em túnel de vento, temse que o ponto ótimo de operação seria para $61,9^{\circ} \mathrm{C}, 0,575 \mathrm{~m} / \mathrm{s}$ e como o tempo de operação não foi significativo em nenhum modelo, tem-se como valor otimizado o mínimo da faixa de operação, 150 minutos.

Figura 2 - RM em função da temperatura e velocidade

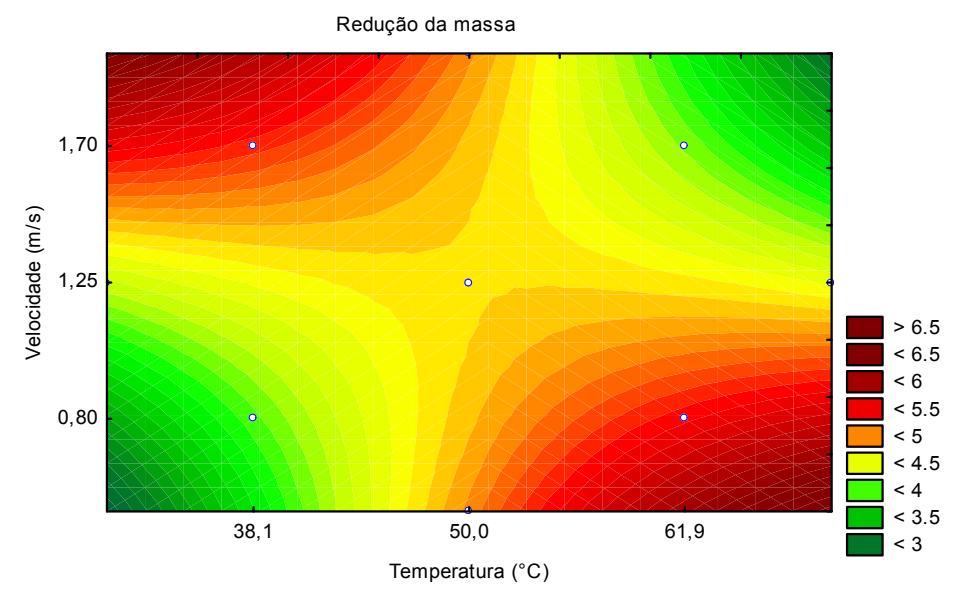

Figura 3 - RAA em função da temperatura codificada 

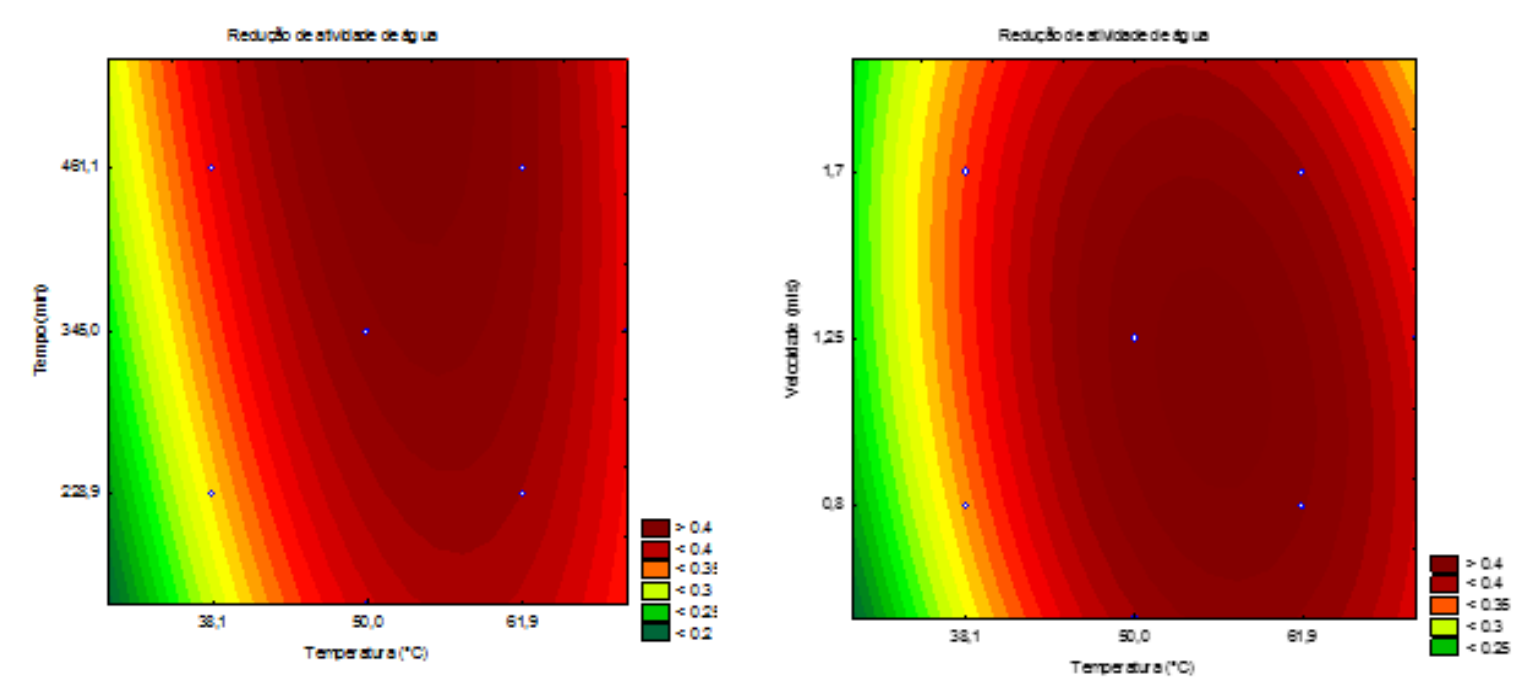

Figura 4 - AAF em função da temperatura e velocidade

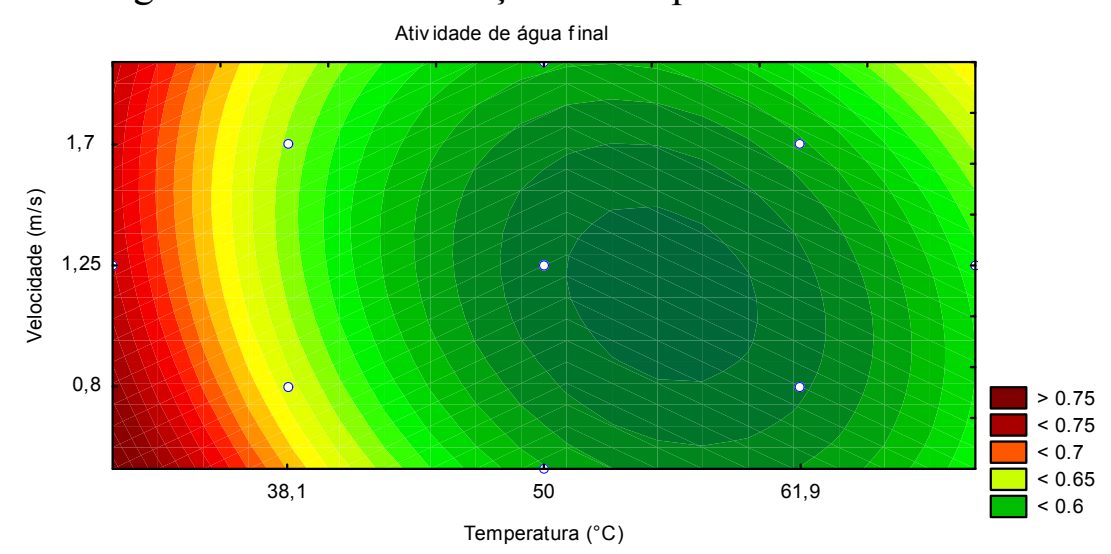

\section{CONCLUSÃO}

Com a secagem em túnel de vento obteve-se a redução da massa e da atividade de água, o que permitiu o alcance dos objetivos da operação unitária, no caso com a redução de tamanho e conservação de alimento. No caso, os modelos apresentaram a interação temperatura, velocidade e tempo e a importância de cada fator para se obter um bom resultado final. Assim, no caso, o ponto otimizado da secagem em túnel foi considerado para uma temperatura de $61,9^{\circ} \mathrm{C}$, velocidade de $0,575 \mathrm{~m} / \mathrm{s}$ e tempo de 150 minutos.

\section{REFERÊNCIAS}

BORGES, S. V. et al. Secagem de fatias de abóboras (Cucurbita moschata,L.) por convecção natural e forçada. Ciência e Tecnologia de Alimentos, Campinas, v.28 (Supl.), p. 245-251, dez., 2008.

ERTEKIN, C.; YALDIZ, O. Drying of eggplant and selection of a suitable thin layer drying model. Journal of Food Engineering., v.63, p.349-359, 2004.

McMINN, W. A. M.; MAGEE, T. R. A. Principles, methods and applications of the convective drying of foodstuffs. Trans. IChemE, v. 77, Part C, set., 1999. 
PARK, K. J. et al. Estudo de secagem de pêra bartlett (Pyrus sp.) em fatias. Ciência e Tecnologia em Alimentos. Campinas, V.21, n.3, p.288-292, set-dez 2001.

PINHEIRO, L. N. et al. Aplicação da desidratação osmótica como pré-tratamento para obtenção de chips de berinjela. In: CONGRESSO BRASILEIRO DE QUÍMICA, n.54, 2014, Natal. Anais do CBQ - Congresso Brasileiro de Química.

RADÜNZ, L. L. et al. Análise da cinética de secagem das folhas de salvia. Rev. Bras. Eng. Agríc. Ambiental, v.14, n.9, p.979-986, 2010.

RAOULT-WACK, A. L. Recent advances in the osmotic dehydration of foods. Trends in food science \& technology, Cambridge, England, V. 5, n. 8, p. 255-260, agosto, 1994.

RODRIGUES, M. I.; IEMMA, A.F. Planejamento de Experimentos \& Otimização de processos. $2^{\mathrm{a}}$ ed. Campinas: Cárita Editora, 2009.

SANTOS, K. A. et al. Composição Química da Berinjela (Solanum Melongena). Boletim do Centrode Pesquisa e Processamento de Alimentos (B.CEPPA). Curitiba, v. 20, n. 2, p. 247-256, julho/dezembro, 2002. 